\title{
SELF-IESTING AS A METHOD OF REDUCING COVID-19 INFECTIONS
}

\author{
Togay Evrin ${ }^{1}$, Luiza Szarpak², Michal Pruc ${ }^{3}(0)$ \\ 'Department of Emergency Medicine, Ufuk University Medical Faculty, Dr Ridvan Ege Education and Research Hospital, Ankara, Turkey \\ ${ }^{2}$ Polonia University, Czestochowa, Poland \\ ${ }^{3}$ Polish Society of Disaster Medicine, Warsaw, Poland
}

KEY WORDS: COVID-19, self-testing, COVID-19 tests

Disaster Emerg Med J 2021; 6(2): 94-95

To the Editor,

At the end of 2019, a new type of coronavirus appeared in China - SARS-CoV-2, which became a challenge for the health care system [1]. Currently, the fight against the third wave of cases is underway all over the world - too few vaccinated people still do not give us the possibility of obtaining herd immunity, which we are trying to get at all costs to fight the pandemic. Here, our activities should focus on infection prevention. The Indian variant of the B.1.617 coronavirus has recently appeared in the world, about which we still know little, and there are more and more questions about the effectiveness of vaccines against this strain. The Indian variant of SARS-CoV-2 shows two important changes to its genome on the protein surface: E484Q and L452R. Single mutations have been known for a long time. The E484Q resembles the E484K variant, which also occurs in the British, South African and Brazilian mutations. The L452R variant is found in the Californian strain of the coronavirus CAL.20C. In the India variant, both mutations occurred for the first time, so we deal with the SARS-CoV-2 double mutation. When an additional strain of the virus escapes our immune system, a so-called escape variant will arise - increased resistance to neutralization by antibodies and T lymphocytes contracting the Indian mutation of the coronavirus.

Here, we should focus on securing and relieving the medical care system in the best possible way. Antigen and PCR tests performed by specialized medical facilities have become commonplace access to which may become difficult because of the increasing number of infections. Here, one should consider self-testing by patients at home or elsewhere - and this has also been introduced in the US, where self-collection kits and testing are available for prescription or over the counter at a pharmacy or retail store [2]. Rapid Antigen Detection Tests (RADT), which can be used as self-tests for the detection of SARS-CoV-2, are also becoming available in the European Union. These tests require individuals to collect a sample, conduct the test, and interpret the results themselves [3].

Many studies have shown that the agreement of results between the self-test and the test performed by healthcare professionals was very similar. Positive per cent agreement between self-testing results and professional RADT testing was $91.4 \%(95 \% \mathrm{Cl}$ 77.6-97.0), while negative per cent agreement was $99.1 \%(95 \% \mathrm{Cl} 95.0-100)$. Although deviations in sampling and testing, i.e., incomplete self-sampling or extraction procedures, or imprecise test performance as volume applied to the test device, have been observed in more than half of the positive samples, it has been found that self-test results may be comparable to those got by qualified medical services [4]. Stohr et al. compared the clinical trial to a home testing situation. 3.215 participants received the BD VeriStor System RADT or RADT self-check kits from Roche Diagnostics and used them on self-collected nasal swab specimens. The sensitivity of the self-tests was compared to gold by the standard method (RT-PCR), which involved taking a sample by a healthcare professional and sending it to a lab- 
oratory for testing. They found the sensitivity to be 75.5\% (95\% Cl: 66.6-82.6) for BD RADT and $80.1 \%$ (95\% Cl: 72.7-86.0) for Roche RADT. Both RADTs showed a very high specificity > 99\% [BD RADT: 99.7\% (95\% Cl: 99.2-99.9); Roche RADT: 99.1\% (95\% Cl: 98.5-99.5)] [5].

Both studies show that self-tests performed by patients are as effective as those performed by trained healthcare professionals. After obtaining the result, the only step that the infected person must take is to contact a doctor or the appropriate institution responsible for entering into the register, which prevents the infected person from contacting the rest of the public and thus further spreading the virus. Most people have COVID-19 disease mildly and can be treated at home. Self-testing significantly relieves testing points of patients and health care facilities - an important issue is also to reduce the risk of infection of people who obtain a negative result without exposing them to the risk of infection at test collection points. Self-testing will also rule out infection in people who have flu or have atypical symp- toms of COVID-19, such as diarrhoea, headaches, or gastrointestinal disorders. From a public health perspective, self-tests can be of benefit when used in addition to professionally performed RADT or RT-PCR tests. They can improve the availability of tests and enable patients to obtain results very quickly, which can aid in the early detection of infectious cases and reduce further transmission within society.

\section{REFERENCES:}

1. Dzieciatkowski T, Szarpak L, Filipiak KJ, et al. COVID-19 challenge for modern medicine. Cardiol J. 2020; 27(2): 175-183, doi: 10.5603/ CJ.a2020.0055, indexed in Pubmed: 32286679.

2. https://www.cdc.gov/coronavirus/2019-ncov/testing/self-testing.html.

3. https://www.ecdc.europa.eu/en/publications-data/considerations-use-self-tests-covid-19-eueea.

4. Lindner A, Nikolai 0, Rohardt C, et al. SARS-CoV-2 patient self-testing with an antigen-detecting rapid test: a head-to-head comparison with professional testing. , doi: 10.1101/2021.01.06.20249009.

5. Stohr J, Zwart VF, Goderski G, et al. Self-testing for the detection of SARS-CoV-2 infection with rapid antigen tests., doi: 10.1101/2021.02.21.21252153 